\title{
Lipidomic profiling reveals distinct differences in plasma lipid composition in overweight or obese adolescent students
}

\section{Ruili Yin}

Beijing Key Laboratory of Diabetes Prevention and Research, Center for Endocrine Metabolic and Immune Disease, Beijing Luhe Hospital

\section{Xiaojing Wang}

Beijing Key Laboratory of Diabetes Prevention and Research, Center for Endocrine Metabolic and Immune Disease, Beijing Luhe Hospital

\section{Kun Li}

Beijing Key Laboratory of Diabetes Prevention and Research, Center for Endocrine Metabolic and Immune Disease, Beijing Luhe Hospital

\section{$\mathrm{Ke} Y u$}

Beijing Key Laboratory of Diabetes Prevention and Research, Center for Endocrine Metabolic and Immune Disease, Beijing Luhe Hospital

\section{Longyan Yang ( $\sim$ lyyang15@ccmu.edu.cn )}

Beijing Key Laboratory of Diabetes Prevention and Research, Center for Endocrine Metabolic and Immune Disease, Beijing Luhe Hospital

\section{Research Article}

Keywords: Lipidomic, Obesity, Overweight, Adolescence

Posted Date: March 9th, 2021

DOI: https://doi.org/10.21203/rs.3.rs-33013/v3

License: @ (i) This work is licensed under a Creative Commons Attribution 4.0 International License. Read Full License 


\section{Abstract \\ Introduction}

: The relationship between dyslipidemia and obesity has been widely reported, but the global lipid profiles associated with the development of obesity still need to be clarified. An investigation into the association between the lipidomic plasma profile and adolescent obesity may provide new insights into the development of obesity.

\section{Methods}

Mass spectrometry coupled with liquid chromatography was applied to detect the global lipidome in the fasting plasma from 90 Chinese adolescents, including 34 obese adolescents, 26 overweight adolescents, and 30 adolescents with a normal body mass index (BMI). All participants underwent anthropometric measurements by using InBody. Clinical biochemical indicators were measured by Cobas Elecsys.

\section{Results}

Both qualitative and quantitative analyses revealed a gradual change in plasma lipid features among obese students, exhibiting characteristics close to overweight students, but differing significantly from normal students. Compared with normal and overweight students, levels of triglyceride (TG), 18-hydroxycortisol, isohumulinone A, and 11-dihydro-12-norneoquassin were up-regulated in the obese group, while phosphatidylcholine (PC), phosphatidylethanolamine (PE), lysoPC (LPC), lysoPE (LPE), and phosphatidylinositol (PI) were significantly downregulated in the obese group. Then, we conducted Venn diagrams and selected 8 significant metabolites from the 3 paired comparisons. Most of the selected features were significantly correlated with the anthropometric measurements.

\section{Conclusions}

This study demonstrated evidence for a relationship between the eight significant metabolites with obese adolescents. These lipid features may provide a basis for evaluating risk and monitoring the development of obesity.

\section{Background}

A high prevalence of obesity and metabolic syndrome can now be observed in both adults and young people. These phenomena affect 380 million children and adolescents worldwide [1]. Childhood obesity has a significant impact on both physical and psychological health [2]. It could lead to metabolic, pulmonary, orthopedic, neurological, cardiovascular, hepatic, and menstrual disorders [3]. WHO defines adolescence as a period of growth and development between the ages of 10 and 19 years after childhood and before adulthood. It is one of the important transition periods in the life cycle and is characterized by a large amount of growth and change, second only to infancy.

It is believed that adolescent and childhood obesity have reached epidemic levels [1], and about $17 \%$ of children are facing obesity problems in the United States [2]. The increased prevalence of overweight and obesity in children 
and adolescents has been observed in several countries, and weight gain is an independent predictor for metabolic syndrome development, although it is not seen in all obese individuals. Metabolic syndrome is defined as the presence of a combination of risk factors for cardiovascular disease and type 2 diabetes, including obesity, dyslipidemia, hypertension and glucose intolerance [4]. The above conditions, although seen more frequently in adults, can manifest at earlier ages $[5,6]$. Therefore, the diagnosis of the possible presence of obesity at early ages, accompanied by control interventions, should have a favorable impact on the health of adult people and the prevention of cardiovascular outcomes.

By comparing the metabolomics characteristics of obesity, Newgard et al. revealed resistance-related BCAA-related metabolite characteristics, and the accompanying specific increase in C3 and C5 carnitine levels, which indicated an increase in BCAA catabolism [7]. Longitudinal lipidomics studies in children have shown that maternal obesity increases the risk of offspring obesity, which is marked by a long-term change in plasma ceramide levels [8]. To study the changes of metabolites in blood lipids by lipidomics, caloric restriction and the improvement of metabolic syndrome following fish oil intake were predicted, and potential lipid metabolites were identified [9]. Pawelzik et al. performed lipidomic analysis of urine samples from obese people and identified a relationship between urinary prostaglandin levels and obesity-related dyslipidemia, abdominal obesity, and insulin resistance [10].

Conventional data-dependent acquisition (DDA) mass spectrometry (MS) mode has been widely used in lipidomic studies, where parameters are detected to minimize duplicate precursor ions and can be optimized to identify complex lipid molecules [11]. However, DDA performance has some inherent limitations, such as a limited dynamic range, a bias against highly abundant ions, and long duty cycles with increasing sample complexity. A data

independent acquisition (DIA) strategy was recently developed to alleviate the limitations of the DDA model [12], which improves detection sensitivity and analytical reproducibility. However, the independent data acquisition method is not easy to apply to lipidomics because the annotation of MS features and the estimation of the false discovery rate in large and complex lipid data sets require more sophisticated software and integrated reference databases [13].

Here, we conducted a non-targeted lipidomic analysis of 90 Chinese adolescent students, including 30 obese students, 26 overweight students, and 34 students with normal BMI, using DIA-based liquid chromatographytandem mass spectrometry (LC-MS/MS). By using statistical business and in-house software to analyze the highly complex data sets, we demonstrated that compared with overweight and normal students, obese students in China have significant changes in lipids in their plasma. In addition, we identified several lipid characteristics, including TG, 18-hydroxycortisol, isohumulinone A, and 11-dihydro-12-norneoquassin, PC, PE, LPC, LPE, and PI, which are potential indicators for predicting obesity risk.

\section{Materials And Methods}

\section{Study population}

Nighty teenagers from junior middle school took part in the study (Beijing 9th Middle School, aged between 12 and 13). In addition, the principal's approval was obtained before visiting the school. During the first visit, a consent form with research information was distributed to the students. We encouraged the students to bring back the consent form the next morning. On the next visit to the school, the children who brought back signed consent forms were screened for inclusion. The volunteers with serious diseases and special diets or recent weight changes 
were excluded. The trial was approved by the Ethics Committee at the Luhe Hospital affiliated with Capital Medical University. All participants with no recent changes in their eating habits or their habits related to physical activity were included. In the research, all procedures complied with the Helsinki Declaration for investigation of human subjects.

\section{Data collection and anthropometric measurement}

The participants underwent anthropometric measurements by using InBody 770 (InBody Co. Ltd., Seoul, Republic of Korea). We evaluated the collected data from the anthropometric measurements statistically and graphically in Microsoft Office Excel 2010 (Los Angeles, CA, USA). In this study, blood was allowed to coagulate at $4^{\circ} \mathrm{C}$ and the serum was separated by centrifugation for $15 \mathrm{~min}$ at 3,000 rpm. Serum TSH, FT4 and FT3 were tested with an electrochemiluminescence immunoassay (ECLIA) using an Abbott Architect I2000 (Abbott Diagnostics, Abbott Park, IL, USA). Clinical biochemical indicators were measured by a Cobas Elecsys 601 (Roche Diagnostics, Switzerland). The children are grouped according to the BMI Z-score of WHO child growth standards [14], and the age is the exact value. Those with a $Z>1$ are defined as overweight group, those with a $Z>2$ are defined as the obese group, and those with a $Z \leq 1$ are defined as normal and thin groups.

To delineate global lipidomic profiles in Chinese overweight and obese adolescents, BMI and body fat percentage together with the corresponding clinical and phenotypic data were collected from the 3 groups in Beijing, China (Additional file 1).

\section{Liquid chromatography-tandem mass spectrometry (LC-MS/MS)}

Lipids were extracted from individual plasma samples and then injected into the mass instrument in both positive and negative modes, with pooled extraction quality control (QC) samples at certain intervals. In this project, the advanced mass spectrometer Xevo G2-XS QTOF (Waters, UK) was used for mass spectrometry data collection, and the commercial software PROGENESIS QI (Version 2.2) (Waters, UK) and the independently developed metabonomics R software package metaX were used for statistical analysis of the mass spectrometry data, wherein metabolite identification was based on the databases HMDB and LipidMaps [15]. Univariate and multivariate analyses were conducted using $\mathrm{R}$ statistics software to identify and evaluate the significant metabolites among the groups [16].

\section{Metabolites extraction method}

First, $40 \mu \mathrm{L}$ of each sample was added to the corresponding 96 -well plate; $120 \mu \mathrm{L}$ of pre-cooled isopropyl alcohol was added, shaken and mixed for $1 \mathrm{~min}$, and then placed at $-20^{\circ} \mathrm{C}$ for $2 \mathrm{~h}$ or overnight, followed by centrifugation at $4000 \mathrm{~g}$ at $4{ }^{\circ} \mathrm{C}$ for $30 \mathrm{~min}$. We placed the supernatant in a new 96 -well plate and diluted it with $225 \mu \mathrm{L}$ of lipid complex solution (isopropanol: acetonitrile: water $=2: 1: 1$ ). Then, $20 \mu \mathrm{L}$ of each sample was mixed with the QC sample and $60 \mu \mathrm{L}$ of the supernatant was transferred to a 96 -well microtiter plate, sealed, and tested on the machine.

\section{Mass spectrometer description}

A high-resolution tandem mass spectrometer Xevo G2 XS QTOF (Waters, UK) was used to detect metabolites eluted from the column. The Q-TOF was operated in both positive and negative ion modes. For positive ion mode, the capillary and sampling cone voltages were set at $3.0 \mathrm{kV}$ and $40.0 \mathrm{~V}$, respectively. For negative ion mode, the capillary and sampling cone voltages were set at $2 \mathrm{kV}$ and $40 \mathrm{~V}$, respectively. The mass spectrometry data were 
acquired in Centroid MSE mode. The TOF mass range was from 100 to $2000 \mathrm{Da}$ in positive mode and 50 to 2000 $\mathrm{Da}$ in negative mode. The survey scan time was $0.2 \mathrm{~s}$. For the MS/MS detection, all precursors were fragmented using $19-45 \mathrm{eV}$, and the scan time was $0.2 \mathrm{~s}$.

\section{Nomenclature of metabolites}

For example, $6.10 \_861.5490 \mathrm{~m} / \mathrm{z}$ was the retention time_ charge mass ratio. The identification results (PC (15:0/0:0), PE (18:0/0:0), LPC (15:0), and LPE (0:0/18:0)) were obtained by comparing the retention time and charge mass ratio information of the collected ions with the information in the KEGG and HMDB databases.

\section{Statistics analysis}

All data was tested with chi-square tests first, then Tukey HSD analysis was applied if it met the normal distribution and the Kruskal-Wallis test was applied if not, and Dunn's post hoc tests followed by pairwise comparisons were performed. Associations between lipid and clinical or anthropometric parameters were determined by Pearson correlation coefficients by GraphPad Prism 7. A P-value $<0.05$ was considered significant.

\section{Results}

\section{Assessment of clinical characteristics and plasma lipidomic features}

The clinical information, including the physiological and anthropometric indicators of the individuals included in this cohort, is summarized in Table 1. The participants were divided into three groups according to their BMI values. The level of SBP, waist-hip ratio, fat mass, body fat percentage and visceral fat area were significantly higher in both overweight and obese individuals than in the control group, with obese participants exhibiting higher values compared with overweight individuals (Kruskal-Wallis test, $\mathrm{P}<0.001$ ).

We evaluated both coverage and reproducibility of the non-targeted lipidomic data on our sample. Using Progenesis QI 2.0 and metaX, the non-targeted metabolomics analysis yielded 51135 positive ion modes (Additional file 2) and 8988 negative ion modes (Additional file 3).

\section{Overweight and obesity-related features}

Because of the observed effects in obese adolescents on the lipid profiles, we performed a blocked Kruskal-Wallis test, using the obese group as the blocking factor, followed by Dunn's hoc test for paired comparisons. As shown in Additional file 4 and 5, 876 positive and 544 negative features were gradually up-regulated among the 3 groups. Also, there were 1081 positive and 353 negative features down-regulated in Additional file 6 and 7. Of these, there are lipids or lipid-like compounds, also including organ-oxygen compounds, amino acids, peptides, and analogs, benzyl alcohols, glycerophospholipids and triacylglycerol. As shown in Fig. 1, paired comparisons revealed that 460 features (290 positive features in Additional file 8 and negative features in Additional file 11) exhibited significant differences between the control and obese group, whereas 231 and 244 features (Additional file 9 and 12 , Additional file 10 and 13 in both positive and negative, respectively) showed obvious differences between the overweight versus control and obese group, respectively $(\mathrm{P}<0.05)$. Of these significantly changed metabolites, we screened out eight (six positive and two negative) metabolites with significant differences in expression among the three groups. The number of variables distinguishing overweight and obesity suggested that changes in a large 
fraction of the lipid profiles in overweight and obesity were shared, implying that compared with the control group, the overweight and obese group share similar metabolites.

To quantify the differential features among the 3 groups, all detected features were assessed using criteria: 1 ) variable importance of the projection $(\mathrm{VIP})>1.0$ estimated by partial least squares discriminant analysis (PLS-DA); 2) fold change in mass intensity $\geq 1.2$ or $\leq 0.83$; 3) $P<0.05$.

\section{Table 1}

Basic characteristics of the three groups in the study. 


\begin{tabular}{|c|c|c|c|c|c|c|c|}
\hline Variables & $\begin{array}{l}\text { Control } \\
(n=30)\end{array}$ & $\begin{array}{l}\text { Overweight } \\
(n=26)\end{array}$ & Obese $(n=34)$ & $\begin{array}{l}P \text { value } \\
b\end{array}$ & $\begin{array}{l}\text { Obese vs } \\
\text { Overweight } \\
\text { c }\end{array}$ & $\begin{array}{l}\text { Obese } \\
\text { vs } \\
\text { Control } \\
\text { c }\end{array}$ & $\begin{array}{l}\text { Overweight } \\
\text { vs Control } \\
\text { c }\end{array}$ \\
\hline $\begin{array}{l}\text { Sex } \\
\text { (female } \\
\%) \text {, no. } \\
(\%)^{a}\end{array}$ & $18(60.00)$ & 16 (53.33) & $14(46.67)$ & 0.594 & -- & -- & -- \\
\hline Age, year & $12.50 \pm 0.51$ & $12.73 \pm 0.45$ & $12.77 \pm 0.47$ & 0.058 & 0.958 & 0.072 & 0.132 \\
\hline $\begin{array}{l}\text { BMI, } \\
\mathrm{Kg} / \mathrm{m}^{2}\end{array}$ & $17.49 \pm 1.41$ & $23.76 \pm 1.00$ & $29.89 \pm 3.17$ & $<0.0001$ & $<0.0001$ & $<0.0001$ & $<0.0001$ \\
\hline $\begin{array}{l}\text { SBP, } \\
\text { mmHg }\end{array}$ & $111.93 \pm 9.77$ & $120.27 \pm 7.18$ & $123.13 \pm 6.23$ & $<0.0001$ & $<0.0001$ & $<0.0001$ & 0.339 \\
\hline $\begin{array}{l}\text { DBP, } \\
\text { mmHg }\end{array}$ & $68.13 \pm 6.77$ & $68.73 \pm 4.68$ & $70.07 \pm 7.10$ & 0.477 & 0.69 & 0.46 & 0.927 \\
\hline $\begin{array}{l}\text { TG, } \\
\mathrm{mmol} / \mathrm{L}\end{array}$ & $0.88 \pm 0.36$ & $1.08 \pm 0.67$ & $1.12 \pm 0.61$ & 0.207 & 0.964 & 0.225 & 0.342 \\
\hline $\begin{array}{l}\mathrm{CHO}, \\
\mathrm{mmol} / \mathrm{L}\end{array}$ & $4.16 \pm 0.76$ & $4.03 \pm 0.80$ & $4.34 \pm 0.77$ & 0.297 & 0.268 & 0.631 & 0.796 \\
\hline $\begin{array}{l}\text { HDL, } \\
\mathrm{mmol} / \mathrm{L}\end{array}$ & $1.39 \pm 0.29$ & $1.24 \pm 0.22$ & $1.22 \pm 0.20$ & 0.015 & 0.885 & 0.018 & 0.061 \\
\hline $\begin{array}{l}\text { LDL, } \\
\text { mmol/L }\end{array}$ & $2.32 \pm 0.53$ & $2.35 \pm 0.61$ & $2.71 \pm 0.66$ & 0.031 & 0.069 & 0.048 & 0.987 \\
\hline $\begin{array}{l}\text { Waist-hip } \\
\text { ratio }\end{array}$ & $0.79 \pm 0.03$ & $0.85 \pm 0.04$ & $0.91 \pm 0.05$ & $<0.0001$ & $<0.0001$ & $<0.0001$ & $<0.0001$ \\
\hline $\begin{array}{l}\text { FBG, } \\
\mathrm{mmol} / \mathrm{L}\end{array}$ & $5.52 \pm 0.37$ & $5.56 \pm 0.42$ & $5.60 \pm 0.42$ & 0.740 & 0.907 & 0.718 & 0.933 \\
\hline $\begin{array}{l}\text { Fat } \\
\text { mass, } \mathrm{Kg}\end{array}$ & $9.00 \pm 3.33$ & $19.70 \pm 4.05$ & $28.82 \pm 6.96$ & $<0.0001$ & $<0.0001$ & $<0.0001$ & $<0.0001$ \\
\hline $\begin{array}{l}\text { Body fat } \\
\text { percent, } \\
\%\end{array}$ & $19.86 \pm 6.06$ & $31.63 \pm 5.44$ & $38.00 \pm 6.60$ & $<0.0001$ & $<0.0001$ & $<0.0001$ & $<0.0001$ \\
\hline $\begin{array}{l}\text { Visceral } \\
\text { fat area, } \\
\mathrm{cm}^{2}\end{array}$ & $38.78 \pm 14.26$ & $88.76 \pm 24.86$ & $138.81 \pm 39.78$ & $<0.0001$ & $<0.0001$ & $<0.0001$ & $<0.0001$ \\
\hline
\end{tabular}

Values are given as mean \pm SD or number of individuals (\%). BMl: body mass index; SBP: systolic pressure; DBP: diastolic pressure; TG: triglyceride; $\mathrm{CHO}$ : cholesterol; HDL: high density lipoprotein; LDL: Low density lipoprotein; FBG: fast blood glucose.

a $P$ value of chi-square test.

b $P$ value of Kruskal-Wallis test.

c P-value of Dunn's post hoc test. 


\section{Comparison between control and overweight, overweight and obese, and control and obese using random forest classifier and ROC curves}

As the qualitative and quantitative analyses revealed significant differences in the metabolites levels among the three groups and indicated a gradual change from control to obese via overweight, we investigated if the metabolites could predict the risk of further obesity development. To assess this possibility, we used a random forest classifier.

As illustrated in Fig. 1, 8 metabolites were generated. The relationships among the three groups were analyzed by the random forest classifier and receiver operating characteristic (ROC) curves. Fig 2A-C shows that the area under the ROC curve (AUC) is $61.90 \%$ (95\% confidence interval $(\mathrm{Cl})=42.00-85.60 \%), 62.80 \%(95 \% \mathrm{Cl}=21.50-86.50 \%$ ), and $74.30 \%(95 \% \mathrm{Cl}=56.00-91.00 \%)$ between control and overweight, overweight and obese, and control and obese in down-regulated both positive and negative ion mode. For up-regulated, the AUC is $59.70 \%(95 \% \mathrm{Cl}=$ $19.50-82.50 \%), 65.40 \%(95 \% \mathrm{Cl}=34.10-75.50 \%)$, and $72.10 \%(95 \% \mathrm{Cl}=49.00-93.50 \%)$ in Fig 2D-F. Together, these results indicate that the lipidomic profiles are regulated in a complex manner during the development of overweight and obesity.

\section{The level of selected metabolites in the control, overweight and obese groups}

As illustrated in Fig. 1, eight metabolites were selected from both positive and negative ion mode lipidomic profiling. The expression of the selected metabolites is shown in Fig 3. Fig. 3A and Fig. 3B indicate that $6.10 \_861.5490 \mathrm{~m} / \mathrm{z}$ and $1.82 \_480.3095 \mathrm{~m} / \mathrm{z}$ in negative ion mode were gradually decreased in the control, overweight and obese groups. Fig. 3D and Fig. 3H exhibit 1.11_396.2412m/z and 10.13_949.7263m/z in selected positive ion mode were gradually increased in the control, overweight and obese groups. However, 4.86_902.5761m/z was gradually decreased in Fig. 3E, 4.84_530.4012n and 4.96_546.3962n peaked in the overweight group (Fig. 3F-G). In summary, the development of obesity may go through the process of overweight in most cases, but it may directly develop into obesity through the alterations of some lipid metabolites.

\section{Correlations between the selected metabolites and clinical parameters}

In the body of overweight and obese people, metabolism is inevitably changed. Hence, the metabolites are also changed. To investigate the relationship between the selected metabolites and clinical parameters, we performed a correlation analysis. As shown in Fig.4A, 6.10_861.5490m/z was negatively correlated with BMI, visceral fat area, body fat percent, and waist/hip ratio. 1.82_480.3095m/z was negatively correlated with BMI, visceral fat area, body fat percent, and waist/hip ratio, but positively correlated with triglyceride in Fig. 4B. 4.84_530.4012n was negatively correlated with total cholesterol (CHO) in Fig. 4C. 1.11_396.2412m/z was positively correlated with BMI, visceral fat area, and waist/hip ratio in Fig. 4D. 4.86_902.5761 m/z was negatively correlated with BMI, but positively with triglyceride (Fig. 4E). 10.13_949.7263m/z was positively correlated with BMI, visceral fat area, waist/hip ratio, triglyceride, and body fat percent (Fig. 4F).

Phospholipids phosphatidylcholine (PC) and phosphatidylethanolamine (PE) are the two most abundant phospholipid species in eukaryotic cells [17]. Lysophosphatidylcholine (LPC), an important signaling molecule and fatty acid carrier, constitutes 5-20\% of total plasma phospholipids [18]. Phosphatidylinositol (PI) plays an important role in cell morphology, metabolic regulation, signal transduction and various physiological functions.

1.82_480.3095m/z was annotated as PC (15:0/0:0), PE (18:0/0:0), LPC (15:0), and LPE (0:0/18:0).

6.10_861.5490m/z was annotated as PI (14:0/22:2(13Z, 16Z))- PI (22:2(13Z,16Z)/14:0) (Additional file 3). 
1.11_396.2412m/z was annotated as 18-hydroxycortisol, isohumulinone A, and 11-dihydro-12-norneoquassin; 4.86_902.5761m/z was annotated as PI (18:0/20:5 (5Z,8Z,11Z,14Z,17Z)); and 10.13_949.7263m/z was annotated as TG $(20: 4(5 Z, 8 Z, 11 Z, 14 Z) / 20: 3(5 Z, 8 Z, 11 Z) / 18: 3(9 Z, 12 Z, 15 Z))$. The levels of TG, 18-hydroxycortisol, isohumulinone $A$, and 11-dihydro-12-norneoquassin were up-regulated in the obese group, while PC, PE, LPC, LPE, and PI were significantly down-regulated in the obese group than in control and overweight individuals (Additional file 2).

\section{Discussion}

Due to the increased prevalence of obesity in children and adolescents, various studies have been conducted to discover which associations and risk factors increase the likelihood of obesity in children. Although it is still difficult to fully grasp all of the risk factors related to obesity, it is of great significance to control and prevent obesity by combining diet, exercise, physiological factors and psychological factors [2]. The short-term and longterm effects of obesity on children's health are a major issue due to adverse psychological and health consequences [19]. Potential negative psychological outcomes are depressive symptoms, poor body image, low self-esteem, risk of eating disorders, and behavioral and learning problems; negative health consequences include insulin resistance, type 2 diabetes, asthma, hypertension, and nonalcoholic steatohepatitis [19, 20]. Obese children are more likely to become obese adults, and therefore increase their risk of multiple diseases before they even reach puberty [20].

The characteristics of human lipomics reflect the early stage of lipid metabolism, including pathophysiological changes related to diseases. Wang et al. observed the levels of five LPC species in an obese group were significantly reduced relative to a normal-weight group [21]. In addition, total LPC, LPC18:0, LPC18:2 and LPC20:4 levels in obese and obese subjects with type 2 diabetes were lower than in nonobese adults. There was no difference in the LPC profile between obese individuals and obese subjects with type 2 diabetes [22]. Moreover, Wallace et al. reported several LPC species were associated with BMI and inflammatory markers [23]. Compared with lean subjects, LPC14:0 and LPC18:0 were higher while LPC18:1 was lower in obese subjects [24].

As we all know, obesity can be estimated by several methods: body mass index (BMI), the ratio of weight to the square of height, is used as the most common indicator of obesity [25]. It is convenient and simple, but it can cause changes in cardiovascular and metabolic performance between individuals. However, there are alternative ways to distribute body fat. A higher WHR indicates more intraperitoneal cavity fat and is associated with a higher risk of type 2 diabetes, cardiovascular disease and mortality [26]. At the same time, waist circumference can also be used. Similar to WHR [27], it is considered a more direct and reliable method. Generally, body fat percentage (BFP) is a method used to measure the ratio of adipose tissue to lean mass and water [28], and is usually determined using bioelectrical impedance. BFP is not related to BMI since it is associated with an increase in allcause mortality, but it is generally suggested to estimate obesity better than BMI [29]. Therefore, this study aimed at Chinese adolescents, a group with a relatively stable diet and lifestyle, carried out a lipidomic study to observe the development process of obesity and to screen out some biochemical indicators for predicting obesity.

In the present study, the levels of TG, 18-hydroxycortisol, isohumulinone A, and 11-dihydro-12-norneoquassin were up-regulated in the obese group, while PC, PE, LPC, LPE, and PI were significantly down-regulated in the obese group relative to the control and overweight individuals. 1.82_480.3095m/z was annotated as PC (15:0/0:0), PE (18:0/0:0), LPC (15:0), and LPE (0:0/18:0). 6.10_861.5490m/z was annotated as PI (14:0/22:2(13Z, 16Z)) - PI $(22: 2(13 Z, 16 Z) / 14: 0)$ (Additional file 3). According to Figure 1, eight metabolites generated only in 
1.11_396.2412m/z were annotated as 18-hydroxycortisol, isohumulinone A, and 11-dihydro-12-norneoquassin; 4.86_902.5761m/Z was annotated as PI (18:0/20:5 (5Z,8Z,11Z,14Z,17Z)); and 10.13_949.7263m/Z was annotated as TG $(20: 4(5 Z, 8 Z, 11 Z, 14 Z) / 20: 3(5 Z, 8 Z, 11 Z) / 18: 3(9 Z, 12 Z, 15 Z))$ (Additional file 2). These data suggest that the development of obesity does not always have to go through an overweight stage, and it may develop directly due to some changes in lipid metabolism.

There are also some limitations to our study. First of all, it was a cross-sectional study that only addressed the alterations of lipidomic profiling in normal, overweight and obese students. Furthermore, the subjects were just grouped according to BMI rather than randomly, and therefore, this may produce selection bias. In addition, this is a small sample study. So based on the above limitations, more large-scale population studies are needed for future investigations.

\section{Conclusions}

In conclusion, this investigation identified eight altered metabolites in Chinese obese and overweight students. These discriminatory metabolites may play important roles in the pathogenesis of obesity and provide a basis for evaluating the risk of and monitoring obesity development.

\section{Declarations}

\section{Ethical Approval and Consent to Participate}

All of the participants signed informed consent for all of the measurements in this research, and the trial was approved by the Ethics Committee at the Beijing Luhe Hospital affiliated with Capital Medical University.

\section{Availability of data and material}

The author has produced the original data described in this manuscript, which can be obtained free of charge by any scientist who wants to use it, without violating the confidentiality rules of the participants.

\section{Funding}

This study was funded by the Beijing Natural Science Foundation (Project Number: 7184222) and National Natural Science Foundation of China (Project Number: 81800768).

\section{Authors' contributions}

R.Y. performed the majority of experiments and drafted the manuscript. X.W. and K.L. helped with experiments and analyzed the data. K.Y. and L.Y. conceived the study, supervised the experiments and revised the manuscript. All authors reviewed the manuscript.

\section{Acknowledgements}

We thank associate professor Dong Zhao at Beijing Luhe Hospital for providing the available conditions to collect the information of all participants.

\section{Conflicts of interest}


The authors declare that they have no conflicts of interest.

\section{Abbreviations}

BMI: body mass index; SBP: systolic pressure; DBP: diastolic pressure; TG: triglyceride; $\mathrm{CHO}$ : cholesterol; HDL: high density lipoprotein; LDL: Low density lipoprotein; FBG: fast blood glucose. PC: phosphatidylcholine; PE: phosphatidylethanolamine; PI: phosphatidylinositol; DDA: data-dependent acquisition; DIA: data-independent acquisition; LC-MS/MS: liquid chromatography-tandem mass spectrometry; MS: mass spectrometry; UPLC-MS: ultra-performance liquid chromatography-mass spectrometry; VIP: variable importance of the projection.

\section{References}

1. Salamonowicz MM, Zalewska A, Maciejczyk M: Oral consequences of obesity and metabolic syndrome in children and adolescents. Dent Med Prob/2019, 56(1):97-104.

2. Sanyaolu A, Okorie C, Qi X, Locke J, Rehman S: Childhood and Adolescent Obesity in the United States: A Public Health Concern. Glob Pediatr Health 2019, 6:2333794X19891305.

3. Sahoo K, Sahoo B, Choudhury AK, Sofi NY, Kumar R, Bhadoria AS: Childhood obesity: causes and consequences. J Family Med Prim Care 2015, 4(2):187-192.

4. Pietrobelli A, Malavolti M, Battistini NC, Fuiano N: Metabolic syndrome: a child is not a small adult. Int $J$ Pediatr Obes 2008, 3 Suppl 1:67-71.

5. Chissini RBC, Kuschnir MC, de Oliveira CL, Giannini DT, Santos B: Cutoff values for HOMA-IR associated with metabolic syndrome in the Study of Cardiovascular Risk in Adolescents (ERICA Study). Nutrition 2019, 71:110608.

6. de Assuncao Bezerra MK, Freese de Carvalho E, Souza Oliveira J, Pessoa Cesse EA, Cabral de Lira PI, Galvao Tenorio Cavalcante J, Sa Leal V: Health promotion initiatives at school related to overweight, insulin resistance, hypertension and dyslipidemia in adolescents: a cross-sectional study in Recife, Brazil. BMC Public Health 2018, 18(1):223.

7. Newgard CB, An J, Bain JR, Muehlbauer MJ, Stevens RD, Lien LF, Haqq AM, Shah SH, Arlotto M, Slentz CA et al: A branched-chain amino acid-related metabolic signature that differentiates obese and lean humans and contributes to insulin resistance. Cell Metab 2009, 9(4):311-326.

8. Leon-Aguilar LF, Croyal M, Ferchaud-Roucher V, Huang F, Marchat LA, Barraza-Villarreal A, Romieu I, Ramakrishnan U, Krempf M, Ouguerram K et al: Maternal obesity leads to long-term altered levels of plasma ceramides in the offspring as revealed by a longitudinal lipidomic study in children. Int J Obes (Lond) 2019, 43(6):1231-1243.

9. Shabrina A, Tung TH, Nguyen NTK, Lee HC, Wu HT, Wang W, Huang SY: n-3 PUFA and caloric restriction diet alters lipidomic profiles in obese men with metabolic syndrome: a preliminary open study. Eur J Nutr 2019.

10. Pawelzik SC, Avignon A, Idborg H, Boegner C, Stanke-Labesque F, Jakobsson PJ, Sultan A, Back M: Urinary prostaglandin D2 and E2 metabolites associate with abdominal obesity, glucose metabolism, and triglycerides in obese subjects. Prostaglandins Other Lipid Mediat 2019, 145:106361.

11. Kofeler HC, Fauland A, Rechberger GN, Trotzmuller M: Mass spectrometry based lipidomics: an overview of technological platforms. Metabolites 2012, 2(1):19-38. 
12. Mapstone M, Cheema AK, Fiandaca MS, Zhong X, Mhyre TR, MacArthur LH, Hall WJ, Fisher SG, Peterson DR, Haley JM et al: Plasma phospholipids identify antecedent memory impairment in older adults. Nat Med 2014, 20(4):415-418.

13. Hu T, Zhang JL: Mass-spectrometry-based lipidomics. J Sep Sci 2018, 41(1):351-372.

14. WHO obesity and overweight fact sheet: https://www.who.int/news-room/fact-sheets/detail/obesity-andoverweight.

15. 1 Wen B, Mei Z, Zeng C, Liu S: metaX: a flexible and comprehensive software for processing metabolomics data. BMC Bioinformatics 2017, 18(1):183.

16. 1 Zhong H, Fang C, Fan Y, Lu Y, Wen B, Ren H, Hou G, Yang F, Xie H, Jie Z et al: Lipidomic profiling reveals distinct differences in plasma lipid composition in healthy, prediabetic, and type 2 diabetic individuals. Gigascience 2017, 6(7):1-12.

17. 1 Gibellini F, Smith TK: The Kennedy pathway-De novo synthesis of phosphatidylethanolamine and phosphatidylcholine. IUBMB Life 2010, 62(6):414-428.

18. 1 Virtanen JA, Cheng KH, Somerharju P: Phospholipid composition of the mammalian red cell membrane can be rationalized by a superlattice model. Proc Natl Acad Sci U S A 1998, 95(9):4964-4969.

19. 1 Skinner AC, Steiner MJ, Henderson FW, Perrin EM: Multiple markers of inflammation and weight status: cross-sectional analyses throughout childhood. Pediatrics 2010, 125(4):e801-809.

20. 2 Lakshman R, Elks CE, Ong KK: Childhood obesity. Circulation 2012, 126(14):1770-1779.

21. 2 Wang Y, Jiang CT, Song JY, Song QY, Ma J, Wang HJ: Lipidomic Profile Revealed the Association of Plasma Lysophosphatidylcholines with Adolescent Obesity. Biomed Res Int 2019, 2019:1382418.

22. 2 Barber MN, Risis S, Yang C, Meikle PJ, Staples M, Febbraio MA, Bruce CR: Plasma lysophosphatidylcholine levels are reduced in obesity and type 2 diabetes. PLOS One 2012, 7(7):e41456.

23. 2 Wallace M, Morris C, O'Grada CM, Ryan M, Dillon ET, Coleman E, Gibney ER, Gibney MJ, Roche HM, Brennan $\mathrm{L}$ : Relationship between the lipidome, inflammatory markers and insulin resistance. Mol Biosyst 2014, 10(6):1586-1595.

24. 2 Kim JY, Park JY, Kim OY, Ham BM, Kim HJ, Kwon DY, Jang Y, Lee JH: Metabolic profiling of plasma in overweight/obese and lean men using ultra performance liquid chromatography and Q-TOF mass spectrometry (UPLC-Q-TOF MS). J Proteome Res 2010, 9(9):4368-4375.

25. 2 Keys A, Fidanza F, Karvonen MJ, Kimura N, Taylor HL: Indices of relative weight and obesity. J Chronic Dis 1972, 25(6):329-343.

26. 2 Pischon T, Boeing H, Hoffmann K, Bergmann M, Schulze MB, Overvad K, van der Schouw YT, Spencer E, Moons KG, Tjonneland A et al: General and abdominal adiposity and risk of death in Europe. N Engl J Med 2008, 359(20):2105-2120.

27. 2 Visscher TL, Seidell JC, Molarius A, van der Kuip D, Hofman A, Witteman JC: A comparison of body mass index, waist-hip ratio and waist circumference as predictors of all-cause mortality among the elderly: the Rotterdam study. Int J Obes Relat Metab Disord 2001, 25(11):1730-1735.

28. 2 Calling S, Hedblad B, Engstrom G, Berglund G, Janzon L: Effects of body fatness and physical activity on cardiovascular risk: risk prediction using the bioelectrical impedance method. Scand J Public Health 2006, 34(6):568-575. 
29. 2 Padwal R, Leslie WD, Lix LM, Majumdar SR: Relationship Among Body Fat Percentage, Body Mass Index, and All-Cause Mortality: A Cohort Study. Ann Intern Med 2016, 164(8):532-541.

\section{Figures}

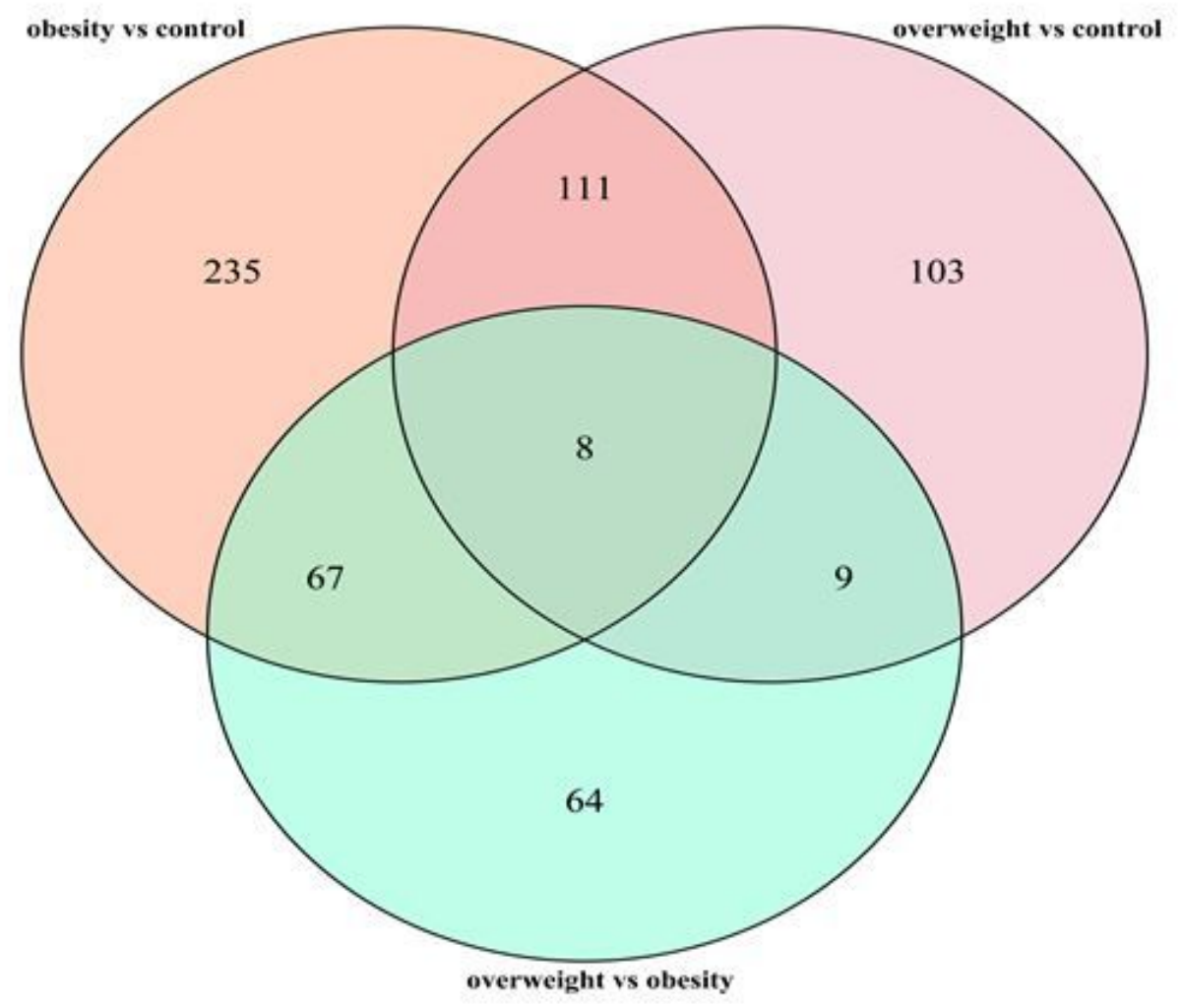

Figure 1

Venn diagram of significant metabolites from the 3 paired comparisons. Venn diagram depicting the number of significant metabolic features from 3 paired comparisons (the direction of change was ignored, $P<0.05$, Dunn's post hoc test). 
A

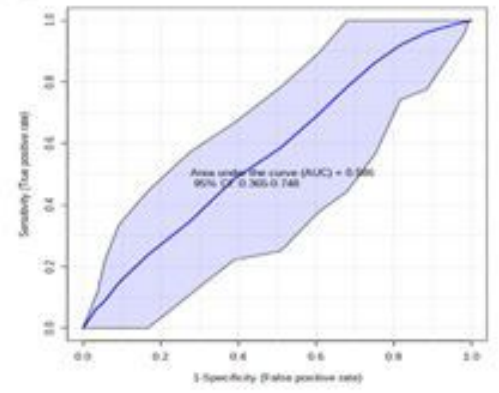

D

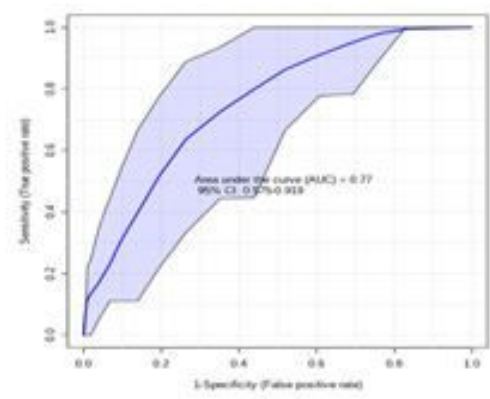

B

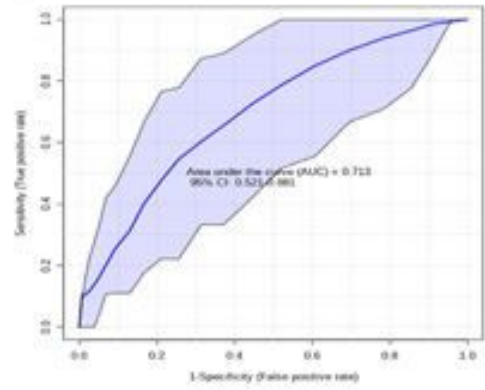

E

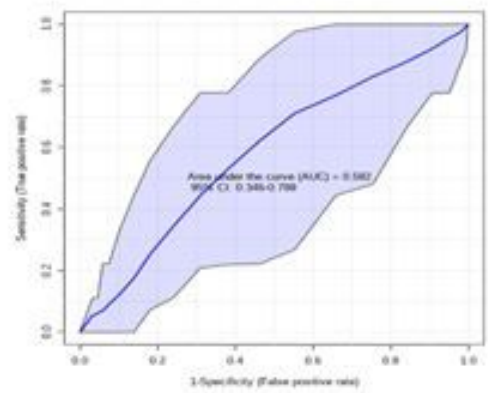

$\mathrm{C}$

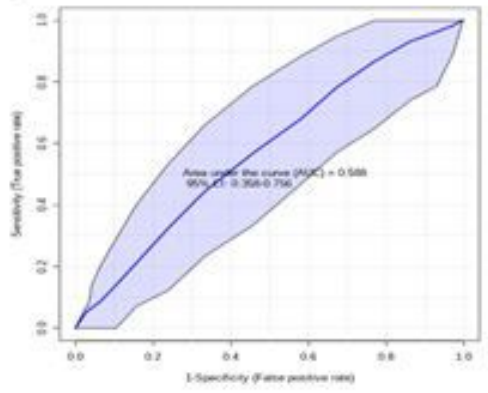

$\mathrm{F}$

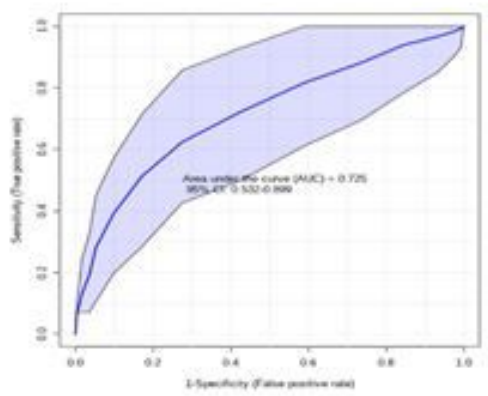

\section{Figure 2}

Receiver operating characteristic curves and areas under the ROC curves in the training set. (A-F) ROC and AUC for the validation set with Control and Overweight, Overweight and Obese, and Control and Overweight, respectively. The model was trained using decreased and increased intensity of the detected features from positive and negative ion mode in the training set among control, overweight and obese $(n=30)$. 
A

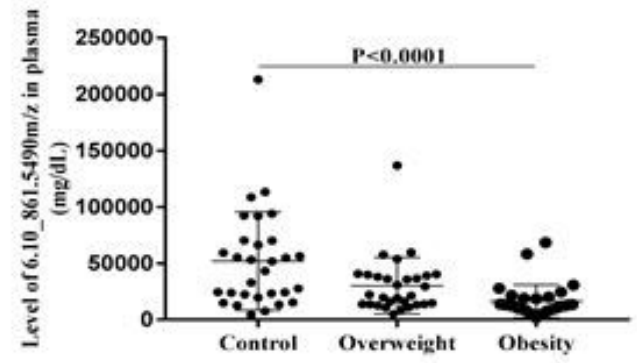

C

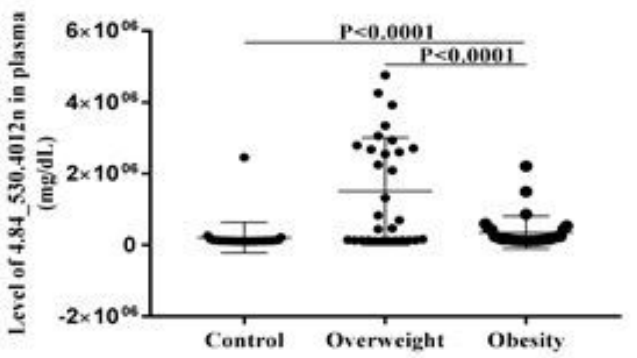

E

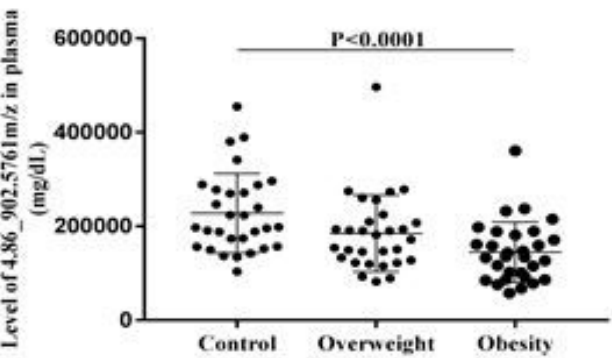

G

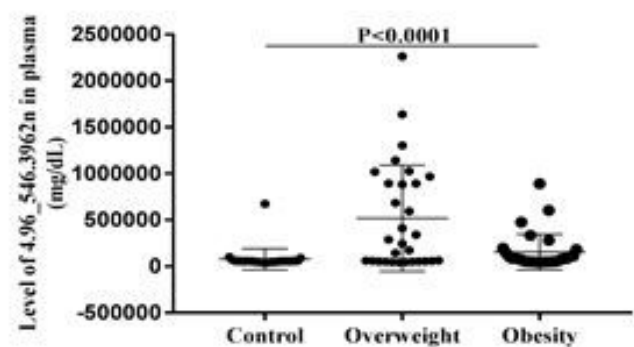

B

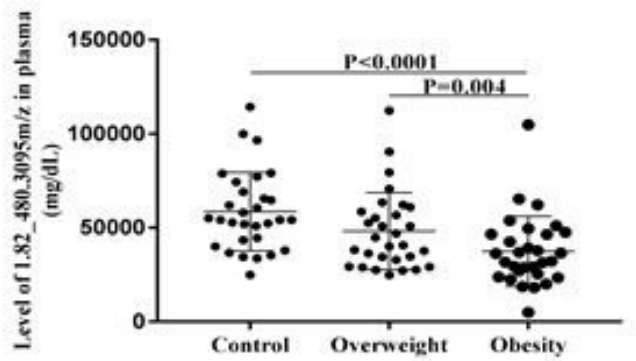

D

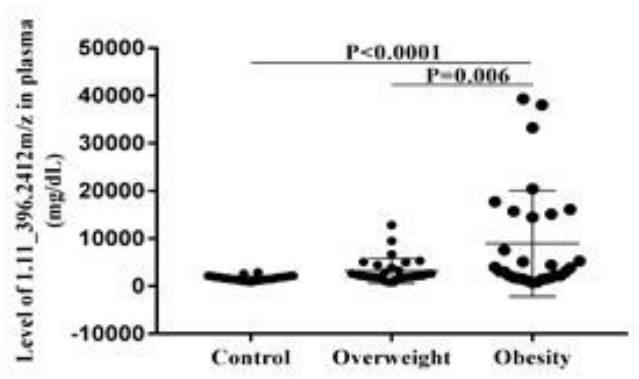

F

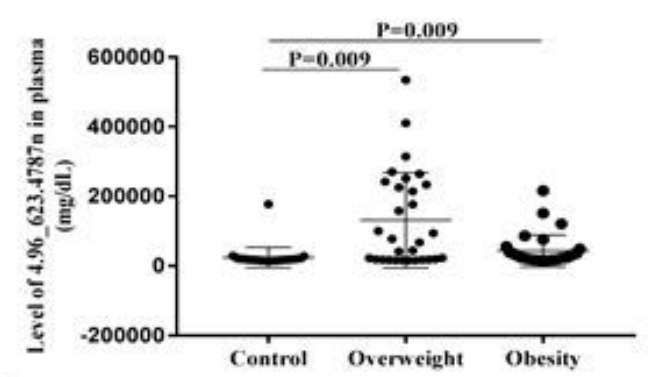

H

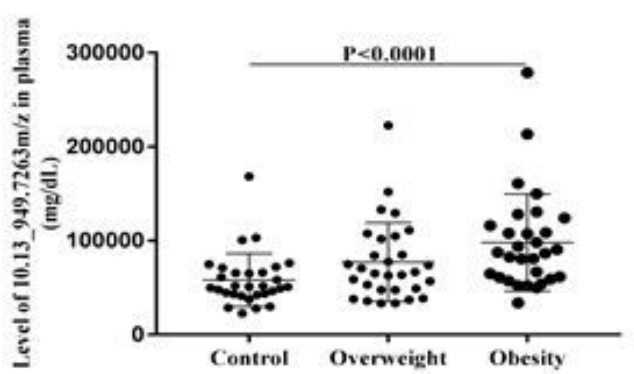

Figure 3

Level of selected metabolites in control, overweight and obese groups. (A) and (B) show negative ion modes level in the control, overweight and obese groups. (C-G) show positive ion modes level in the control, overweight and obese groups. 
A

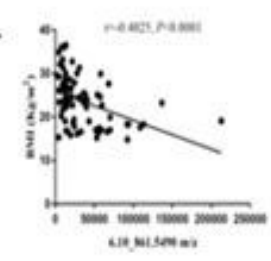

B

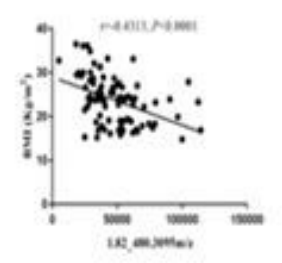

C

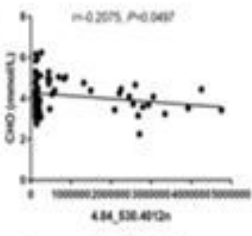

D

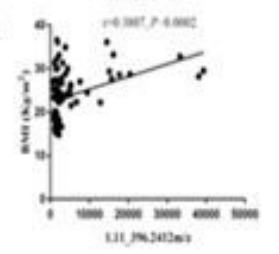

$\mathbf{E}$

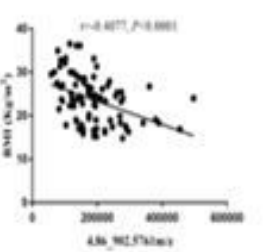

F

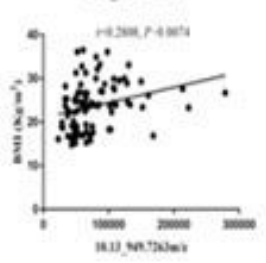

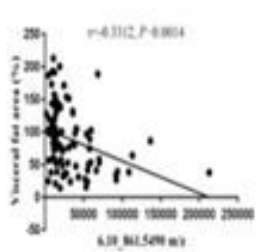
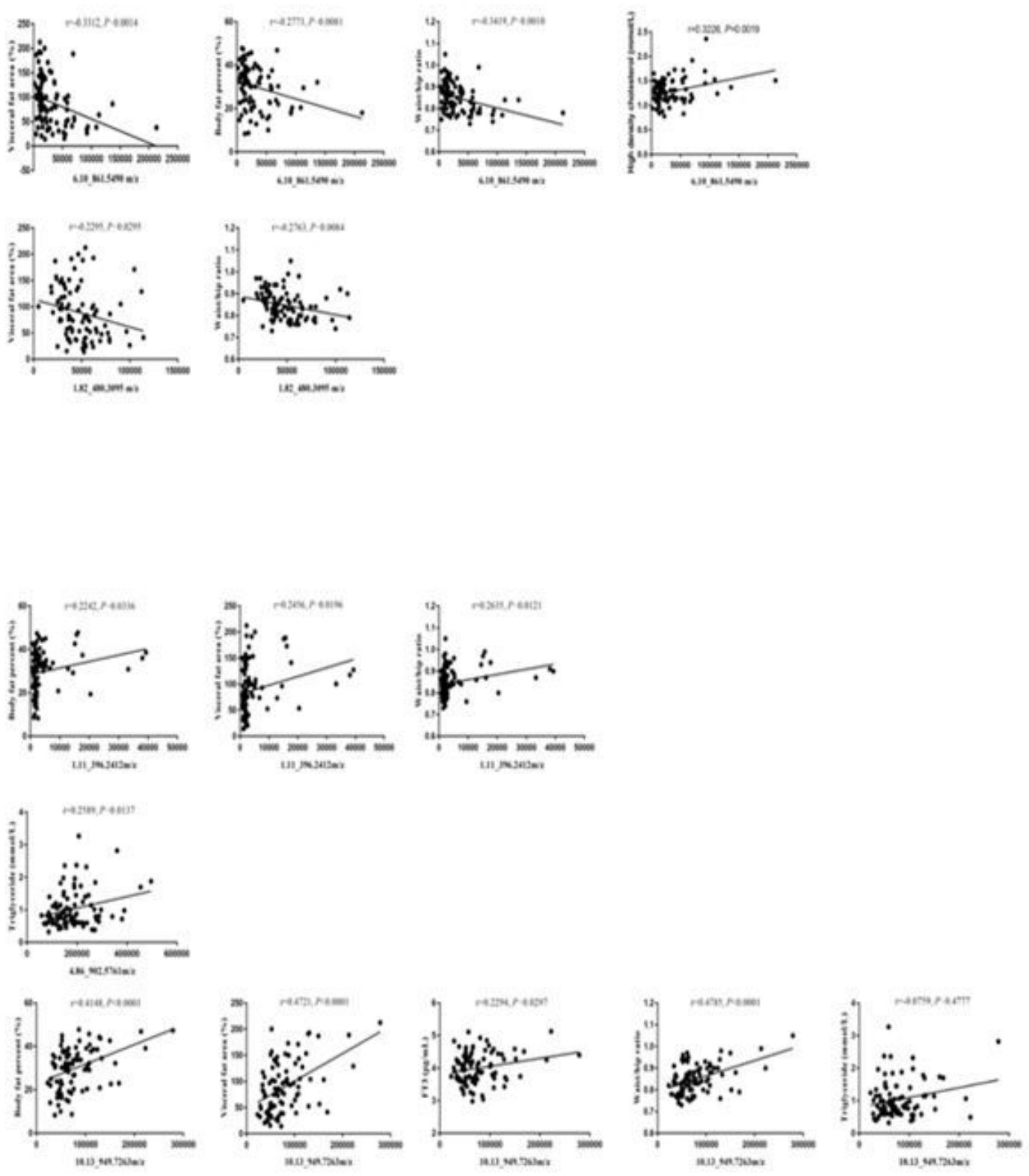

\section{Figure 4}

Correlation between clinical parameters and selected features. (A) 6.10_861.5490m/z was negatively correlated with BMI, visceral fat area, body fat percent, waist/hip ratio, and HDL. (B) 1.82_480.3095m/z was negatively correlated with BMI, visceral fat area, body fat percent, and waist/hip ratio. (C) 4.84_530.4012n was negatively correlated with cholesterol. (D) 1.11_396.2412m/z was positively correlated with BMI, visceral fat area, body fat percent, and waist/hip ratio. (E) 4.86_902.5761 m/z was negatively correlated with BMI, but positively with triglyceride. (F) 10.13_949.7263m/z was positively correlated with BMI, body fat percent, visceral fat area, FT3, waist/hip ratio, and triglyceride.

\section{Supplementary Files}

This is a list of supplementary files associated with this preprint. Click to download. 
- Additionalfile1.xls

- Additionalfile8obesityBcontroldiffheatmappos.xls

- Additionalfile1.xls

- Additionalfile13obesityAobesityBdiffheatmapneg.xls

- Additionalfile11obesityBcontroldiffheatmapneg.xls

- Additionalfile10obesityAobesityBdiffheatmappos.xls

- Additionalfile5.xls

- Additionalfile11obesityBcontroldiffheatmapneg.xls

- Additionalfile12obesityAcontroldiffheatmapneg.xls

- Additionalfile12obesityAcontroldiffheatmapneg.xls

- Additionalfile9obesityAcontroldiffheatmappos.xls

- Additionalfile13obesityAobesityBdiffheatmapneg.xls

- Additionalfile10obesityAobesityBdiffheatmappos.xls

- Additionalfile3metaboAnalystInputneg.xls

- Additionalfile4.xls

- Additionalfile4.xls

- Additionalfile5.xls

- Additionalfile7.xls

- Additionalfile6.xls

- Additionalfile6.xls

- Additionalfile3metaboAnalystlnputneg.xls

- Additionalfile7.xls

- Supplementarytables.docx

- Additionalfile8obesityBcontroldiffheatmappos.xls

- Additionalfile9obesityAcontroldiffheatmappos.xls

- Supplementarytables.docx 